ARTICLE

Received 27 Oct 2016 | Accepted 23 Mar 2017 | Published 26 May $2017 \quad$ DOl: 10.1038/ncomms15376

\title{
Scalable focused ion beam creation of nearly lifetime-limited single quantum emitters in diamond nanostructures
}

Tim Schröder ${ }^{1, \star \uparrow}$, , Matthew E. Trusheim ${ }^{1, \star}$, Michael Walsh ${ }^{1, \star}$, Luozhou Li ${ }^{1}$, Jiabao Zheng ${ }^{1, \dagger}$, Marco Schukraft ${ }^{1}$, Alp Sipahigil ${ }^{2}$, Ruffin E. Evans ${ }^{2}$, Denis D. Sukachev ${ }^{2} \dagger$, Christian T. Nguyen $^{2}$, Jose L. Pacheco ${ }^{3}$, Ryan M. Camacho ${ }^{3}$, Edward S. Bielejec ${ }^{3}$, Mikhail D. Lukin ${ }^{2} \&$ Dirk Englund ${ }^{1}$

The controlled creation of defect centre-nanocavity systems is one of the outstanding challenges for efficiently interfacing spin quantum memories with photons for photon-based entanglement operations in a quantum network. Here we demonstrate direct, maskless creation of atom-like single silicon vacancy (SiV) centres in diamond nanostructures via focused ion beam implantation with $\sim 32 \mathrm{~nm}$ lateral precision and $<50 \mathrm{~nm}$ positioning accuracy relative to a nanocavity. We determine the $\mathrm{Si}+$ ion to $\mathrm{SiV}$ centre conversion yield to be $\sim 2.5 \%$ and observe a 10 -fold conversion yield increase by additional electron irradiation. Low-temperature spectroscopy reveals inhomogeneously broadened ensemble emission linewidths of $\sim 51 \mathrm{GHz}$ and close to lifetime-limited single-emitter transition linewidths down to $126 \pm 13 \mathrm{MHz}$ corresponding to $\sim 1.4$ times the natural linewidth. This method for the targeted generation of nearly transform-limited quantum emitters should facilitate the development of scalable solid-state quantum information processors.

\footnotetext{
${ }^{1}$ Department of Electrical Engineering and Computer Science, Massachusetts Institute of Technology, Cambridge, Massachusetts 02139, USA.

2 Department of Physics, Harvard University, 17 Oxford Street, Cambridge, Massachusetts 02138, USA. ${ }^{3}$ Sandia National Laboratories, Albuquerque, New Mexico 87185, USA. * These authors contributed equally to this work. † Present addresses: Niels Bohr Institute, University of Copenhagen, Denmark (T.S.); Columbia University, New York, New York 10027, USA (J.Z.); Russian Quantum Center and P.N. Lebedev Physical Institute, Moscow 143025, Russia (D.D.S.). Correspondence and requests for materials should be addressed to T.S. (email: schroder@nbi.ku.dk) or to D.E. (email: englund@mit.edu).
} 
A central goal in semiconductor quantum optics is to devise efficient interfaces between photons and atom-like quantum emitters for applications including quantum memories, single-photon sources and nonlinearities at the level of single quanta. Many approaches have been investigated for positioning emitters relative to the mode-maximum of nanophotonic devices with the necessary subwavelength-scale precision, including fabrication of nanostructures around pre-localized or site-controlled semiconductor quantum dots ${ }^{1-5}$ or diamond defect centres ${ }^{6}$, or implantation of ions for defect centre creation in nanostructures concomitant with the nanofabrication ${ }^{7,8}$. However, these approaches have not allowed high-throughput post-fabrication creation of quantum emitters with nearly indistinguishable emission in nanophotonic structures already fabricated and evaluated; such an approach greatly simplifies the design and fabrication process and improves the yield of coupled emitter-nanostructure systems.

Unlike quantum emitters such as molecules or quantum dots, diamond defect centres can be created through ion implantation and subsequent annealing 9,10 , enabling direct control of the centre depth via the ion energy. Lateral control has been demonstrated through the use of nanofabricated implantation masks ${ }^{11-16}$, which have been employed for colour centre creation relative to optical structures through atomic force microscope (AFM) mask alignment ${ }^{6}$, and combined implantation/nanostructure masking ${ }^{7,8}$. Implantation through a pierced AFM tip ${ }^{6}$ does not require modification of the fabrication process and allows for implantation after fabrication and evaluation of these structures. However, the process is time-consuming, requires special AFM tips and can lead to reduced positioning precision by collisions with mask walls. As an alternative, focused ion beam (FIB) implantation of ions, for example, nitrogen ${ }^{17}$ and silicon ${ }^{18}$, can greatly simplify the implantation process by eliminating the need of a nanofabricated mask. Similar to a scanning electron microscope, an ion beam can be precisely scanned, enabling lateral positioning accuracy at the nanometre scale and 'direct writing' into tens of thousands of structures with high throughput.

The silicon vacancy (SiV) belongs to a group of colour centres in diamond that has emerged as promising single-photon emitters and spin-based quantum memories. Among the many diamond-based fluorescent defects that have been investigated ${ }^{19}$, the SiV centre ${ }^{20-23}$ is exceptional in generating nearly lifetimelimited photons with a high Debye-Waller factor of 0.79 (ref. 24) and low spectral diffusion due to a vanishing permanent electric dipole moment in an unstrained lattice 25,26 . These favourable optical properties have notably enabled two-photon quantum interference between distant $\mathrm{SiV}$ centres ${ }^{25,27}$ and entanglement of two $\mathrm{SiV}$ centres coupled to the same waveguide ${ }^{28}$. In addition, the $\mathrm{SiV}$ has electronic and nuclear spin degrees of freedom that could enable long-lived, optically accessible quantum memories ${ }^{29-31}$.

Here we introduce a method for positioning emitters relative to the mode-maximum of nanophotonic devices: direct FIB implantation of $\mathrm{Si}$ ions into diamond photonic structures. This post-fabrication approach to quantum emitter generation achieves nanometre-scale positioning accuracy and creates SiV centres with optical transition linewidths comparable to the best 'naturally' growth-incorporated $\mathrm{SiV}$ reported ${ }^{27}$. The approach allows Si implantation into $\sim 2 \times 10^{4}$ sites per second, which allows creation of millions of emitters across a wafer-scale sample. We also show that additional post-implantation electron irradiation and annealing creates an order of magnitude enhancement in Si to SiV conversion yield. By repeated cycles of Si implantation and optical characterization, this approach promises nanostructures with precisely one $\mathrm{SiV}$ emitter per desired location. Finally, we demonstrate and evaluate the site-targeted creation of $\mathrm{SiVs}$ in pre-fabricated diamond photonic crystal nanocavities. The ability to implant quantum emitters with high spatial resolution and yield opens the door to the reliable fabrication of efficient light-matter interfaces based on semiconductor defects coupled to nanophotonic devices.

\section{Results}

Spatial precision of SiV creation. As outlined in Fig. 1, the fabrication approach introduced here relies on Si implantation in a custom-built $100 \mathrm{kV}$ FIB nanoImplanter (A\&D FIB100nI) system (Methods) and subsequent high-temperature annealing to create $\mathrm{SiV}$ centres. The nanoImplanter uses field emission to create a tightly focused ion beam down to a minimum spot size of $<10 \mathrm{~nm}$ from a variety of liquid metal alloy ion sources (Methods). For the experiments described here, we used an Si beam with a typical spot size of $<40 \mathrm{~nm}$ into commercially available high-purity chemical vapour deposition diamond substrates (Element6). After implantation, we performed hightemperature annealing and surface preparation steps to convert implanted Si ions to SiVs (Methods).
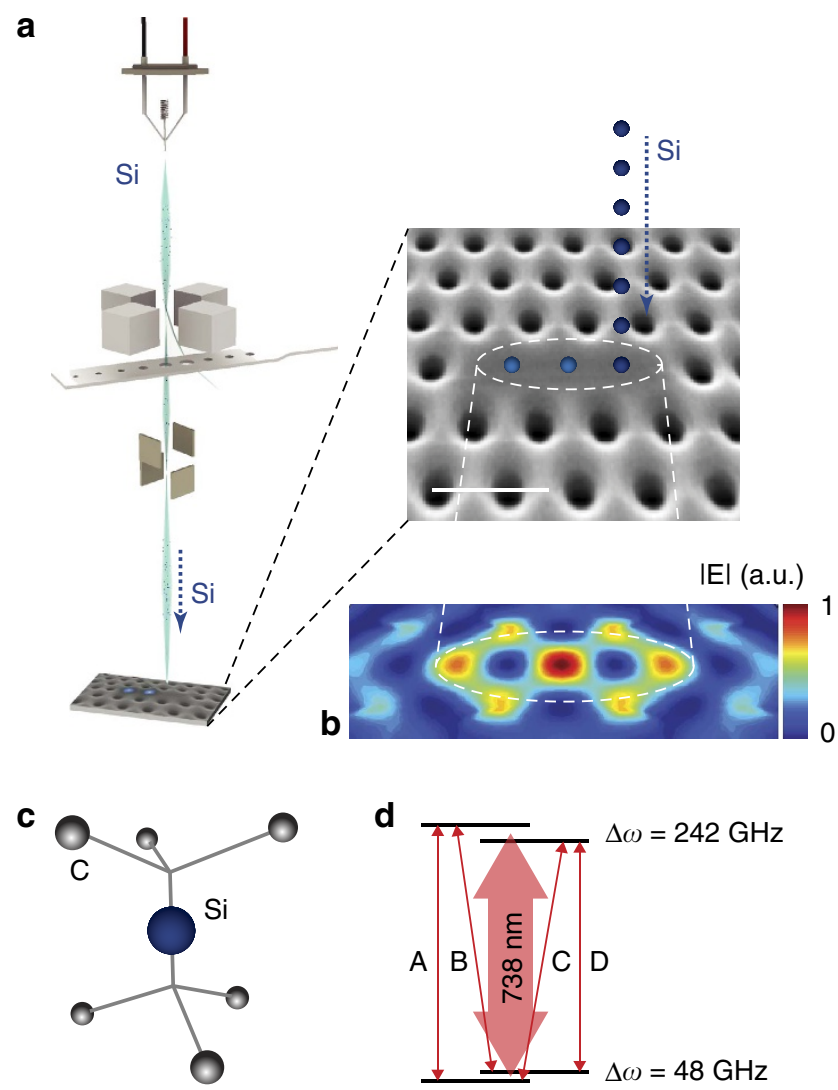

Figure 1 | Targeted Si ion implantation into diamond and SiV defect properties. (a) Illustration of targeted ion implantation. Si ions are precisely positioned into diamond nanostructures via a FIB. The zoom-in shows a scanning electron micrograph of a $\mathrm{L} 3$ photonic crystal cavity patterned into a diamond thin film. Scale bar, $500 \mathrm{~nm}$; Si is silicon. (b) Intensity distribution of the fundamental L3 cavity mode with three Si target positions: the three mode-maxima along the centre of the cavity are indicated by the dashed circle. The central mode peak is the global maximum. (c) Atomic structure of a SiV defect centre in diamond. Si represents an interstitial Si atom between a split vacancy along the $<111>$ lattice orientation and $C$ the diamond lattice carbon atoms. (d) Simplified energy-level diagram of the negatively charged SiV indicating the four main transitions $A, B, C$ and $D^{26} . \Delta \omega$ is the energy splitting of the two levels within the doublets. 

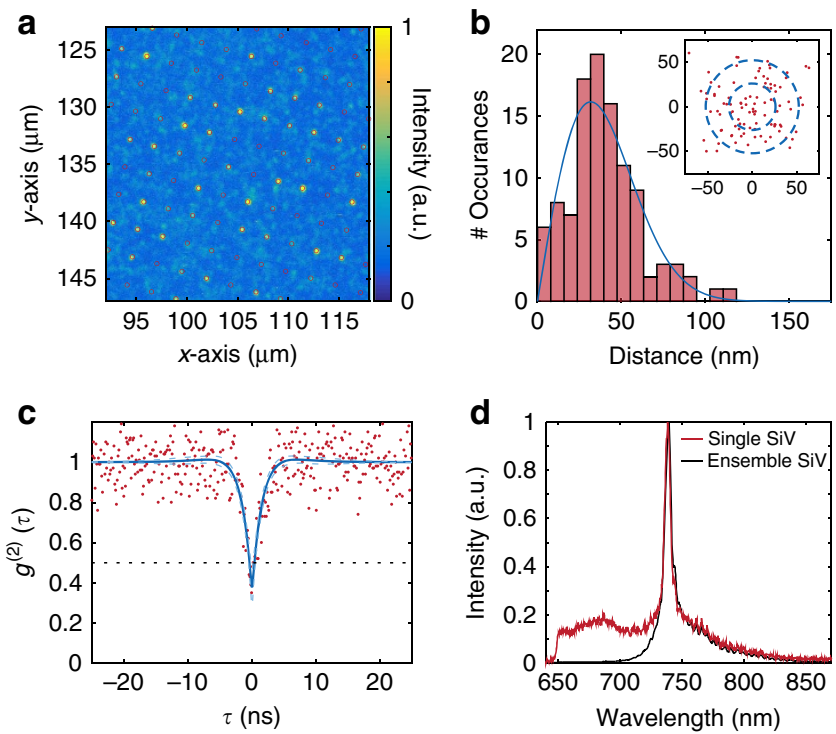

Figure 2 I Spatial precision of SiV creation. (a) Confocal scan of SiV centre array. Sites are separated by $2.14 \mu \mathrm{m}$. Overlaid are regular grid points from an aberration-corrected reference lattice. (b) Analysis of implantation precision. We determine the 2D position uncertainty of the created SiV be $40 \pm 20 \mathrm{~nm}$. Blue curve: fit to Rayleigh distribution. Inset: Scatter plot of created single SiV sites relative to their grid points with one and two $\sigma$ guides to the eye, where the radius $\sigma=26 \mathrm{~nm}$ corresponds to the expected implantation s.d. resulting from the combination of beam width and implant straggle. (c) Normalized second-order autocorrelation function of a single SiV with $g^{(2)}(0)=0.38 \pm 0.09$. Red points indicate data (without background subtraction), and the blue line is a fit to the function $1-A \cdot \exp \left(-\left|\tau / t_{1}\right|\right)+B \cdot \exp \left(-\left|\tau / t_{2}\right|\right)$. The black dashed line indicates $g^{(2)}(\tau)=0.5$, while the blue dashed lines indicate the $95 \%$ confidence interval on the fit. (d) Ensemble (black) and single-emitter (red) SiV room temperature fluorescence spectra. The characteristic zero-phonon line at $737 \mathrm{~nm}$ is prominent.

We characterized the resulting $\mathrm{SiV}$ arrays at room temperature through confocal fluorescence microscopy in a home-built set-up (Methods). Figure 2a shows a scan of a square array of $\mathrm{SiV}$ implantation sites with lattice spacing of $2.14 \mu \mathrm{m}$ across a $30 \times 30 \mu \mathrm{m}^{2}$ write field, created via a single-point exposure from the Si beam. Room temperature spectral measurements in a dense region containing many centres (Fig. $2 \mathrm{~d}$, blue curve) showed a zero-phonon line (ZPL) with an inhomogeneous linewidth of $\sim 5 \mathrm{~nm}$ centred around $738.3 \mathrm{~nm}$, characteristic of the $\mathrm{SiV}$ centre. We subsequently identified single SiVs through second-order correlation measurements. For instance, Fig. 2c shows photon antibunching for a $\mathrm{SiV}$ with an observed count rate of $30 \mathrm{kcts} \mathrm{s}^{-1}$ collected via an oil immersion (numerical aperture (NA) of 1.3) objective into a single-mode fibre under $20 \mathrm{~mW}$ of $532 \mathrm{~nm}$ pump power. The red line in Fig. $2 \mathrm{~d}$ shows the single-emitter fluorescence spectrum at room temperature, which is very similar in shape and linewidth to the inhomogeneous spectrum. At room temperature, these lines are broadened by phonon processes and not limited by inhomogeneity between different SiV centres ${ }^{32}$.

To determine the spatial precision of creating $\mathrm{SiV}$ with our method, we analysed their distribution relative to the implantation lattice grid. Figure $2 \mathrm{~b}$ shows the distance of each imaged implanted single $\mathrm{SiV}$ from the ideal lattice site, resulting in a $\chi$-distribution with a mean separation in $\mathrm{R}$ of $\sigma=40 \pm 20 \mathrm{~nm}$ and underlying lateral $(x, y)$ distributions with zero mean and s.d.'s of $32 \mathrm{~nm}$. These measured values agree well with the expected precision of $26 \mathrm{~nm}$ calculated by the addition in quadrature of the a
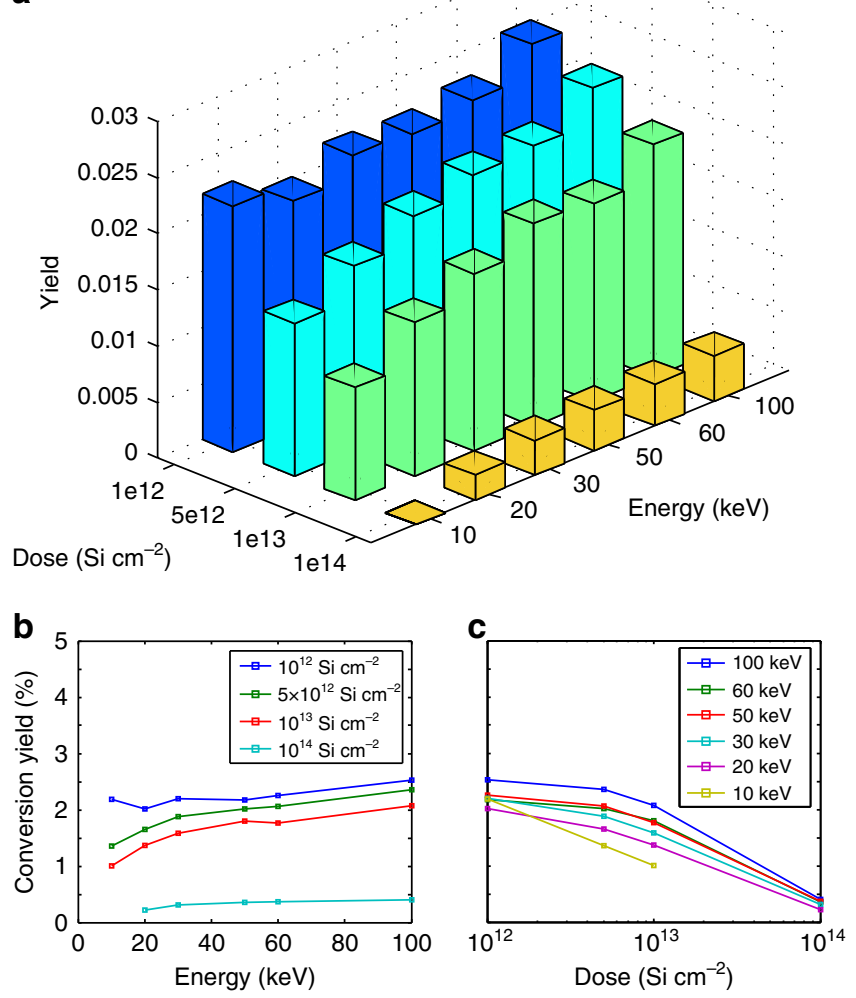

Figure 3 | Si ion to SiV conversion yield. (a) Si to SiV conversion yield for varying implantation ion energies and doses. The conversion yield was determined by calibrating array intensities (Fig. 2) with the determined averaged single SiV photon count rate. Si conversion yield as function of (b) energy and (c) dose. The lines are guides to the eye.

uncertainties arising from the nominal $40 \mathrm{~nm}$ FHWM beam size and $19 \mathrm{~nm}$ lateral implantation straggle.

Creation yield of SiV. To determine the conversion yield of implanted $\mathrm{Si}$ ions to $\mathrm{SiV}$ centres, we swept the implantation dose logarithmically from $10^{12}$ to $10^{14} \mathrm{Si} \mathrm{cm}^{-2}$, and the implantation energy linearly from 10 to $100 \mathrm{keV}$. The dose and energy determine the number and depth of vacancies created during the implantation process, with increased energy resulting in more vacancies at increased depth. The vacancy density affects the probability that a Si defect captures a diffusing vacancy and converts to $\mathrm{SiV}$ during annealing, which is a proposed mechanism for $\mathrm{SiV}$ formation ${ }^{33}$. To estimate the yield, we measured the fluorescence intensity across a $10 \times 10 \mu \mathrm{m}^{2}$ region of constant implantation dose and energy, and normalized to the average single-emitter intensity and implanted ion number. Figure $3 \mathrm{a}$ summarizes the yield measurements. Yield increases as a function of energy (Fig. 3b), which is expected for a vacancy-limited $\mathrm{SiV}$ creation process, up to $2.5 \%$ for the highest-energy $100 \mathrm{keV}$ ions with a dose of $\sim 10^{12} \mathrm{~cm}^{-2}$. These measurements indicated a decreasing yield as a function of dose (Fig. 3c). We attribute this to an accumulation of charged defects in the diamond lattice that lead to ionization, similarly to what was observed in nitrogen vacancy $(\mathrm{NV})$ centres $^{34}$. Alternatively, reduced yield could result from lattice damage that accumulates in the form of multivacancy defects as the diamond lattice approaches the graphitization threshold, a phenomenon that has been observed in similar experiments with NV centres ${ }^{35}$. 


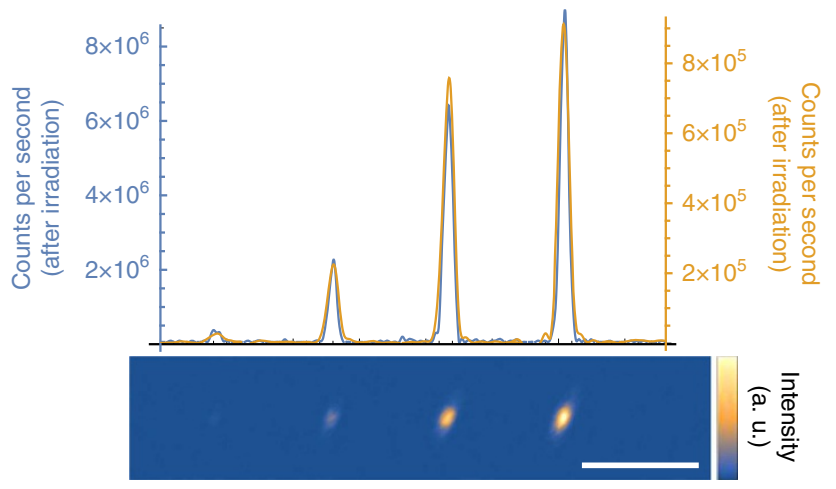

Figure 4 | Electron co-implantation. After electron irradiation and subsequent annealing we observe a 10-fold increase in fluorescence intensity at the implantation positions of $\mathrm{Si}$ ions (lower inset). The $\mathrm{Si}$ ion doses were 500, 2,000, 5,000 and 10,000 ions per spot. The yellow line plot through the fluorescence maximum of the image indicates the intensity before electron irradiation, and the blue line after irradiation. Scale bar, $5 \mu \mathrm{m}$.

Irradiating diamond with high-energy electrons can also improve the conversion yield of vacancy-related colour centres $^{36,37}$. Electron irradiation at high energies $>170 \mathrm{keV}$ (ref. 38) can displace carbon atoms and create additional vacancies, which allows for larger conversion efficiency of implanted ions into vacancy-related colour centres. To verify these experiments with the $\mathrm{SiV}$ centre, we first created a reference sample by implanting four spots with silicon ions in increasing doses of 500,2,000, 5,000 and 10,000 ions per spot into bulk diamond with an implantation energy of $100 \mathrm{keV}$, corresponding to an implantation depth of $\sim 68 \mathrm{~nm}$. After annealing this sample at $1,200^{\circ} \mathrm{C}$ to activate $\mathrm{SiVs}^{39}$, a scanning confocal fluorescence image was taken by exciting these spots simultaneously with $\sim 10 \mathrm{~mW}$ of both $520 \mathrm{~nm}$ (Thorlabs LP520-SF15) and $700 \mathrm{~nm}$ (Thorlabs LP705-SF15) laser light, and collecting light into a single-mode fibre through a $10 \mathrm{~nm}$ bandpass filter (Semrock FF01-740/13) around $737 \mathrm{~nm}$ (Fig. 4, yellow line). After this reference measurement, we irradiated the sample with $1.5 \mathrm{MeV}$ electrons with a total fluence of $\sim 10^{17} \mathrm{~cm}^{-2}$. After another annealing step, a second fluorescence image was taken with the same set-up and it was verified by spectral measurements (Horiba iHR 550 with Synapse CCD) that indeed only the SiV typical peak at $737 \mathrm{~nm}$ was detected. In the second measurement, we observed increased fluorescence for all four spots by a factor of $\sim 10$ (Fig. 4, blue line), corresponding to a final conversion yield of $\sim 20 \%$. This result is consistent with previous observations in Si-doped diamond samples ${ }^{33}$, supporting our interpretation that the conversion efficiency of FIB implantation is limited by the vacancy density in the diamond.

Optical and coherence properties of $\mathrm{SiV}$ at cryogenic temperatures. We next describe the implanted SiV centres' low-temperature spectral properties. Photoluminescence spectral measurements were performed in a home-built confocal cryostat set-up (Methods). The inhomogeneous distribution of SiV transitions at $18 \mathrm{~K}$ is plotted in Fig. 5a with a full width at half maximum (FWHM) of $\sim 0.642 \mathrm{~nm}(\sim 51 \mathrm{GHz})$. We then performed photoluminescence excitation (PLE) measurements determine the linewidths of individual SiVs below the spectrometer limit (Methods). We determined an average single-emitter transition linewidth of $200 \pm 15 \mathrm{MHz}$ from a sample of $10 \mathrm{SiV}$ implanted at $100 \mathrm{keV}$ with individually resolvable transitions. The narrowest observed transition, shown in Fig. 5b, had a linewidth of
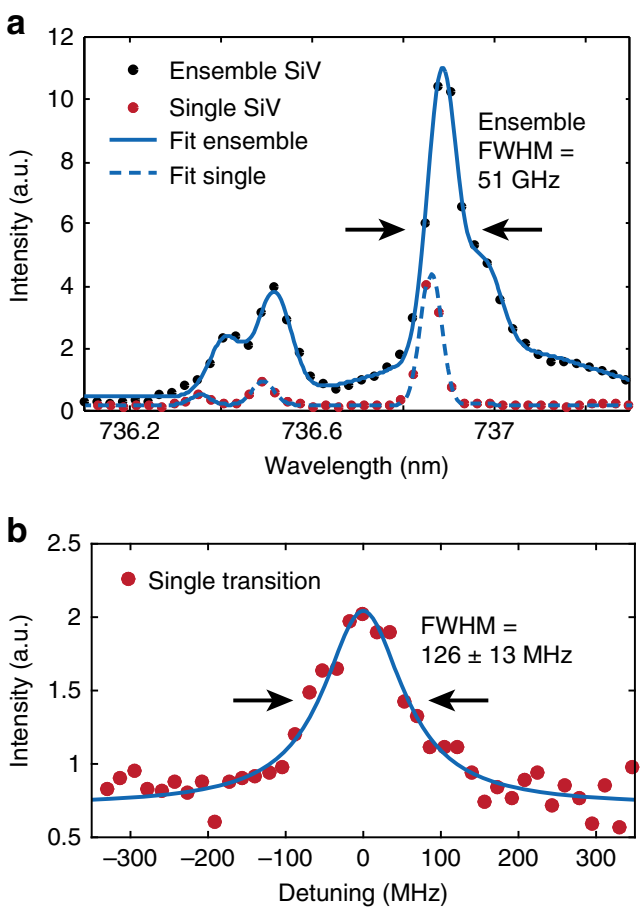

Figure 5 | Optical linewidth and coherence properties of SiV.

(a) Cryogenic spectra ( $<18 \mathrm{~K}$ ) of a single SiV (red circles) and an ensemble (black circles). The four SiV transitions (Fig. 1) as well as the phonon sideband are each fitted with a Gaussian function. The single SiV linewidths are spectrometer-limited (FWHM $=\sim 34 \mathrm{GHz}$ ). For the ensemble, we determine an inhomogeneous broadening as low as $\sim 51 \mathrm{GHz}$ (FWHM). The wavelength values are slightly blue-shifted because of an offset relative to an absolute wavelength reference by $\sim 0.1 \mathrm{~nm}$. (b) Cryogenic (4K) photoluminescence excitation measurement of the narrowest observed single SiV transition with a linewidth of $126 \pm 13 \mathrm{MHz}$ (FWHM, error estimation: $95 \%$ confidence interval) determined with a Lorentzian fit function. This linewidth of an implanted SiV is equal, within error, to the narrowest natural SiV linewidth measured to date.

$126 \pm 13 \mathrm{MHz}$, which is within a factor of 1.4 of the lifetime limit $\gamma=(2 \pi \times 1.7 \mathrm{~ns})^{-1}=94 \mathrm{MHz}$ for a typical fluorescence lifetime of $1.7 \mathrm{~ns}$ (ref. 25), and equivalent to the narrowest lines observed in natural SiVs to date $\mathrm{d}^{27,40}$.

Direct SiV creation in an optical nanocavity. Finally, we demonstrated the targeted implantation and subsequent creation of $\mathrm{SiV}$ centres inside diamond nanostructures. We first fabricated two-dimensional (2D) photonic crystal nanocavities into a 200-nm-thick diamond membrane through oxygen reactive ion etching ${ }^{41,42}$. We then used the FIB system to target $\mathrm{Si}$ ions into the mode-maxima of the photonic crystal cavities. In the case of L3 cavities, we targeted the three mode-maxima individually (Fig. 1b). The Si ion beam was aligned to the cavity through secondary-electron imaging of pre-fabricated alignment markers on the sample (Fig. 6b, Methods). We targeted the cavity mode-maxima with $160 \mathrm{keV}$ ions for an average of $1.8 \mathrm{SiVs}$ per cavity (Methods). After performing the processing steps described in Methods, we observed about one SiV per cavity implantation spot with spectrometer-limited $(<34 \mathrm{GHz})$ ZPL linewidths. To determine the position of a single $\mathrm{SiV}$ relative to the cavity, we performed a spectrally resolved photoluminescence confocal scan (Fig. 6d,e). This measurement allows comparison between the photonic crystal cavity location, determined by Raman scattering, and the SiV location, determined from the emitted fluorescence at the SiV ZPL. By fitting the measured emission patterns to $2 \mathrm{D}$ 


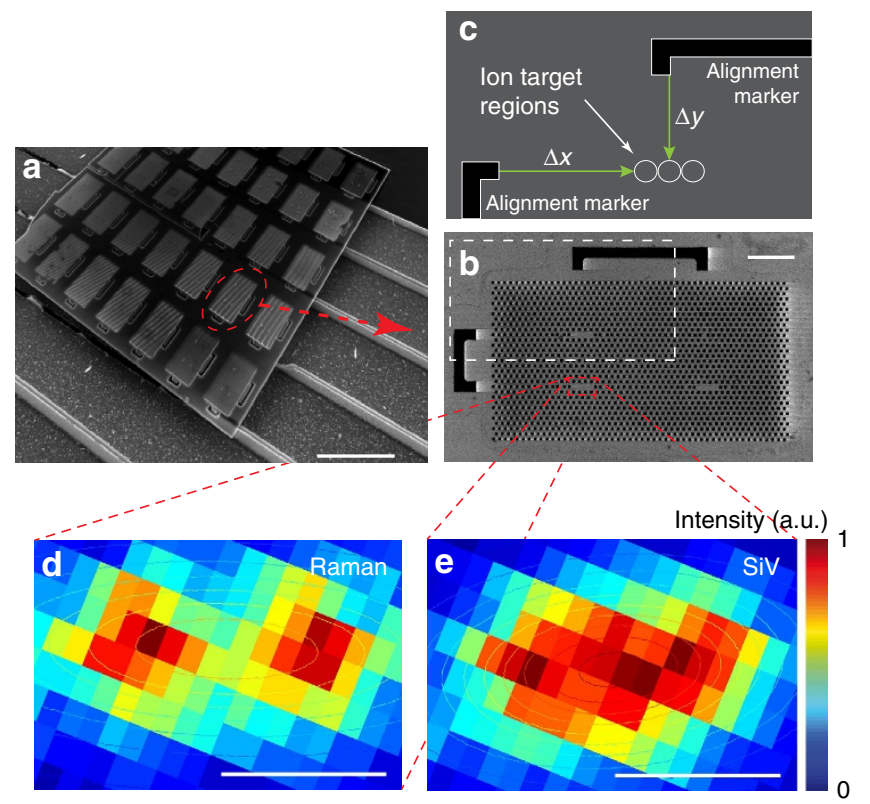

Figure 6 | SiV creation in photonic nanocavity. (a) SEM of example PhC cavity sample. Scale bar, $10 \mu \mathrm{m}$. (b) Close-up SEM of example PhC lattice with four cavities. The white dashed rectangle indicates the area illustrated in c. Scale bar, $2 \mu \mathrm{m}$. (c) Illustration of targeting relative to alignment markers (black) with an ion spot size down to $<40 \mathrm{~nm}$. The white circles (not to scale for visibility) indicate the three L3 cavity mode-maxima (Fig. 1). To determine the SiV-positioning accuracy relative to the mode-maxima, we performed spectrally resolving fluorescence scans. At each pixel in $\mathbf{d}, \mathbf{e}$, we recorded a spectrum including the Raman signal (d), the SiV fluorescence (e) and the cavity resonances (not displayed).

(d) Intensity $x-y$ plot of the diamond Raman signal at $572.8 \mathrm{~nm}$.

(e) Intensity $x-y$ plot of SiV emission at $736.9 \mathrm{~nm}$. By fitting a 2D Gaussian function to the intensity distribution, we determined the distance between the centre of the cavity and the SiV fluorescence, the effective positioning accuracy, to $48(21) \mathrm{nm}$, with error estimation of one s.d. Scale bar in $\mathbf{d}, \mathbf{e}, 0.5 \mu \mathrm{m}$

Gaussians, we estimate a relative positioning accuracy of $48 \pm 21 \mathrm{~nm}$. This is close to the limit set by the combination of beam size and implantation straggle $(\sim 52 \mathrm{~nm})$, with very low offset of $4 \mathrm{~nm}$ in the $x$ direction and a main offset of $48 \mathrm{~nm}$ in the $y$ direction.

\section{Discussion}

While we have demonstrated targeted creation of high-quality SiVs through FIB, there are several avenues for improvement. The stochastic nature of the $\mathrm{SiV}$ creation process, characterized by a mean yield of $\eta$, prevents the generation of exactly one emitter with high yield ${ }^{43}$. One solution is to implant a low dose of $\mathrm{Si}$ ions (to create $\ll 1 \mathrm{SiV}$ on average) and optically verify whether a SiV resulted after annealing. Because of the ability to select implantation sites individually, the FIB process allows for such repeated low-yield implantation steps conditionally halted on the creation of the desired emitter number. An alternative approach to create precisely one quantum emitter is to implant only one ion at a time, as was recently demonstrated ${ }^{44}$, combined with electron irradiation or co-implantation of other ion species to create vacancies ${ }^{34}$ to drive the $\mathrm{SiV}$ conversion yield to unity.

The linewidths of the $\mathrm{SiVs}$ were measured in areas with $2.5 \mathrm{SiVs}$ on average, distributed within only $\sim 55.4 \mathrm{~nm}$ (FWHM) diameter, corresponding to an implantation dose of $\sim 10^{12} \mathrm{~cm}^{-2}$, indicating that high densities of implanted $\mathrm{SiVs}$ are not detrimental for their optical properties. Although we found that FIB-implanted SiVs are similar in homogeneous transition linewidth to 'natural', as-grown centres, the inhomogeneous linewidth of $\sim 51 \mathrm{GHz}$ (after $1,050^{\circ} \mathrm{C}$ annealing) is still slightly broader than the $\sim 15 \mathrm{GHz}$ demonstrated for a similar $\mathrm{SiV}$ creation method with annealing temperatures around $1,200^{\circ} \mathrm{C}$ (ref. 40). Potential causes include di-vacancy break down caused by higher temperatures, or near-surface strain and defects in the diamond due to polishing, which can be reduced by etching the damaged layer before implantation ${ }^{45}$.

In summary, we demonstrated $\mathrm{SiV}$ creation with high spatial accuracy by FIB implantation of $\mathrm{Si}$ ions into bulk and nanostructured diamond. The $\mathrm{SiV}$-positioning accuracy relative to the targeted nanocavity mode-maximum was $48 \pm 21 \mathrm{~nm}$, which is sufficiently precise to locate the SiV within $\sim 90 \%$ of the mode-field intensity maximum of nanocavities or waveguides. We also demonstrated that the $\mathrm{SiV}$ creation yield can be increased after implantation by a factor of 10 , up to $20 \%$. The targeted implantation technique demonstrated here likely applies to other quantum emitters like the germanium defect centre in diamond ${ }^{46}$ and other materials of interest, such as silicon carbide ${ }^{47}$ or molybdenum disulfide; this would be particularly advantageous for materials where traditional nanofabricated masking is challenging.

The ZPLs of SiVs created by our method have optical linewidths within a factor of 1.4 of the lifetime limit, making them as narrow as naturally occurring SiVs described to date. Considering both this narrow linewidth and the narrow inhomogeneous distribution of implanted SiV of only $\sim 51 \mathrm{GHz}$, this fabrication method represents a significant step towards the high-yield generation of thousands to millions of efficiently waveguide-coupled indistinguishable single-photon sources. Such arrays of atom-like quantum emitters would be of great utility for a range of proposed quantum technologies, including quantum networks and modular quantum computing ${ }^{48,49}$, linear optics quantum computing ${ }^{50,51}$, all-photonic quantum repeaters ${ }^{52,53}$ and photonic Boson sampling ${ }^{54}$.

\section{Methods}

Silicon ion implantation. Focused ion implantation was performed at the Ion Beam Laboratory at Sandia National Laboratories using the nanoImplanter (nI). The $\mathrm{nI}$ is a $100 \mathrm{kV}$ FIB machine (A\&D FIB100nI) making use of a three-lens system designed for high mass resolution, using an ExB filter, and single ion implantation, using fast beam blanking. The ExB mass-filter $(M / \Delta M$ of $\sim 61)$ separates out different ionic species and charge states from liquid metal alloy ion sources, providing the capability for implantation of $\sim 1 / 3$ the periodic table over a range of energies from 10 to $200 \mathrm{keV}$. For the $\mathrm{Si}$ implantation discussed here, we used an AuSbSi liquid metal alloy ion source with typical Si beam currents ranging from 0.4 to $1 \mathrm{pA}$. Fast beam blanking allows direct control over the number of implanted ions. We determine the number of implanted ions by measuring the beam current and setting the pulse length to target a given number of ions per pulse. The $\mathrm{nI}$ is a direct write lithography platform that uses electrostatic draw deflectors, controlled by a Raith Elphy Plus pattern generator, to position the beam. Single ion positioning is limited by the beam spot size on target. With typical spot sizes ranging from 10 to $50 \mathrm{~nm}$, we have measured the targeting accuracy to be $<35 \mathrm{~nm}$ for $200 \mathrm{keV} \mathrm{Si}++$ beam using a series of ion beam-induced charge measurements.

For targeting into nanostructures, we align the ion beam relative to the sample by registering a secondary-electron image of the alignment markers generated using the ion beam to scan the sample. Shift, rotation and magnification corrections are calculated and applied in the pattern generator control package. This allows for any location within the write field to be individually targeted for implantation.

The lithography pattern is the original design file that was used to pattern the diamond thin film via electron beam lithography and reactive ion etching. Errors resulting from inaccuracy during electron beam lithography were not taken into account.

To create a single $\mathrm{SiV}$ per cavity with high probability, we implanted $\sim 20 \mathrm{Si}$ ions per cavity mode-maximum, yielding $\sim 1.8 \mathrm{SiVs}$ per cavity on average according to an extrapolated conversion efficiency of $\sim 3 \%$ under Poisson statistics for $160 \mathrm{keVSi}$ ions (Fig. 3) that target the middle of membrane at $106 \mathrm{~nm}$. 
SiV creation and sample preparation. We annealed the sample at $1,050^{\circ} \mathrm{C}$ under high vacuum $\left(<10^{-6}\right.$ mbar at maximum temperature) for $2 \mathrm{~h}$ to form $\mathrm{SiV}$ centres and eliminate other vacancy-related defects. Finally, we clean the sample surface through boiling tri-acid treatment (1:1:1 nitric:perchloric:sulfuric) and subsequent dry oxidation in a $30 \%$ oxygen atmosphere at $450{ }^{\circ} \mathrm{C}$ for $4 \mathrm{~h}$.

Room temperature measurement set-up. We used a modified fluorescence microscope (Zeiss Axio Observer), customized to allow confocal illumination at $532 \mathrm{~nm}$ (Coherent Verdi) and single-mode fibre fluorescence collection. Collected fluorescence is spectrally filtered (Thorlabs FEL0650) and detected on avalanche photodiodes with single-photon resolution (Excelitas) or spectrally resolved on a grating spectrometer (Princeton Instruments, Acton SP2500i).

Cryogenic measurement set-up. These measurements were performed at $18 \mathrm{~K}$ in a closed cycle helium cryostat (Janis). A home-built confocal microscope collects the fluorescence with a high NA objective (Olympus UMplanfl $100 \times 0.95$ NA) and directs the emission to either the input of a single-mode fibre connected to an avalanche photodiode or to a free-space spectrometer with a resolution of $\sim 61 \mathrm{pm}$ $(\sim 34 \mathrm{GHz})$ at $737 \mathrm{~nm}$ (Princeton Instruments, IsoPlane SCT 320).

Photoluminescence excitation measurements were performed using a modified helium flow probe-station (Desert Cryogenics model PS-100) with a $0.95 \mathrm{NA}$ microscope objective (Nikon CFI LU Plan Apo Epi $100 \times$ ) inside the vacuum chamber. Details of this set-up are described in ref. 40.

Analysis of spatial positioning precision. To determine the spatial precision of the SiV implantation, we created and imaged a square array of SiV colour centres following the procedures in Methods A and D. We then fitted each SiV site with a 2D Gaussian to determine the location of the SiV centres below the diffraction limit, and considered only SiV sites with fluorescence intensities consistent with single emitters. Using these locations, we fitted a $2 \mathrm{D}$ grid allowing for affine transformation and found the distance between each $\mathrm{SiV}$ site and its nearest grid point. Finally, we binned the distances and fitted to a central $\gamma$-distribution with two degrees of freedom (Rayleigh distribution), which describes the distribution of the distance $R=\sqrt{X^{2}+Y^{2}}$ where $X$ and $Y$ are independent zero mean normal random variables with identical variance (Fig. $2 b$ ). The reported separation is the mean of the fitted $\chi$-distrbution corresponding to the mean separation in $\mathrm{R}$ $(40 \mathrm{~nm})$, and the error is the square root of the variance $(20 \mathrm{~nm})$. The mean separation in the $X$ and $Y$ directions is $0 \mathrm{~nm}$ with a s.d. of $32 \mathrm{~nm}$.

Analysis of targeted implantation accuracy. To determine the positioning accuracy of the cavity-targeted SiV creation, we performed a spectrally resolved photoluminescence confocal scan at room temperature. At each pixel of a 2D $532 \mathrm{~nm}$ laser scan we recorded a spectrum and determined the intensity for different spectral positions. For each wavelength, we then plotted its 2D-intensity map as in Fig. 6d,e. This measurement allows comparison between the photonic crystal location, determined by Raman scattering of the $532 \mathrm{~nm}$ laser pump from the diamond $(572.52 \mathrm{~nm})$, which is present in the cavity region but not in the surrounding air holes, and the SiV location determined from the emitted fluorescence (at $736.98 \mathrm{~nm}$ ). By fitting the measured emission patterns to $2 \mathrm{~d}$ Gaussians, we estimate a relative positioning accuracy of $48(21) \mathrm{nm}$. The error is estimated from the $68 \%$ fitting confidence interval, which corresponds to one s.d.

Data availability. The data that support the findings of this study are available from the corresponding author.

\section{References}

1. Badolato, A. et al. Deterministic coupling of single quantum dots to single nanocavity modes. Science 308, 1158-1161 (2005).

2. Sapienza, L., Davanço, M., Badolato, A. \& Srinivasan, K. Nanoscale optical positioning of single quantum dots for bright and pure single-photon emission. Nat. Commun. 6, 7833 (2015).

3. Schneider, C. et al. Microcavity enhanced single photon emission from an electrically driven site-controlled quantum dot. Appl. Phys. Lett. 100, 091108 (2012).

4. Birindelli, S. et al. Single photons on demand from novel site-controlled GaAsN/GaAsN:H quantum dots. Nano Lett. 14, 1275-1280 (2014).

5. Surrente, A. et al. Polarization properties and disorder effects in $\mathrm{H} 3$ photonic crystal cavities incorporating site-controlled, high-symmetry quantum dot arrays. Appl. Phys. Lett. 107, 031106 (2015).

6. Riedrich-Möller, J. et al. Nanoimplantation and Purcell enhancement of single nitrogen-vacancy centers in photonic crystal cavities in diamond. Appl. Phys. Lett. 106, 221103 (2015).

7. Schröder, T. et al. in Conference on Lasers and Electro-Optics 2014, FW1B.6 (OSA Technical Digest, 2014).

8. Schukraft, M. et al. Invited article: precision nanoimplantation of nitrogen vacancy centers into diamond photonic crystal cavities and waveguides. APL Photon. 1, 020801 (2016).
9. Meijer, J. et al. Generation of single color centers by focused nitrogen implantation. Appl. Phys. Lett. 87, 261909 (2005).

10. Rabeau, J. R. et al. Implantation of labelled single nitrogen vacancy centers in diamond using N15. Appl. Phys. Lett. 88, 023113 (2006).

11. Naydenov, B. et al. Engineering single photon emitters by ion implantation in diamond. Appl. Phys. Lett. 95, 181109-181109-3 (2009).

12. Toyli, D. M., Weis, C. D., Fuchs, G. D., Schenkel, T. \& Awschalom, D. D. Chip-scale nanofabrication of single spins and spin arrays in diamond. Nano Lett. 10, 3168-3172 (2010).

13. Pezzagna, S. et al. Nanoscale engineering and optical addressing of single spins in diamond. Small 6, 2117-2121 (2010).

14. Spinicelli, P. et al. Engineered arrays of nitrogen-vacancy color centers in diamond based on implantation of $\mathrm{CN}$ - molecules through nanoapertures. New. J. Phys. 13, 025014 (2011).

15. Pezzagna, S., Rogalla, D., Wildanger, D., Meijer, J. \& Zaitsev, A. Creation and nature of optical centres in diamond for single-photon emission-overview and critical remarks. New J. Phys. 13, 035024 (2011).

16. Bayn, I. et al. Generation of ensembles of individually resolvable nitrogen vacancies using nanometer-scale apertures in ultrahigh-aspect ratio planar implantation masks. Nano Lett. 15, 1751-1758 (2015).

17. Lesik, M. et al. Maskless and targeted creation of arrays of colour centres in diamond using focused ion beam technology. Phys. Status Solid. (A) 210, 2055-2059 (2013).

18. Tamura, S. et al. Array of bright silicon-vacancy centers in diamond fabricated by low-energy focused ion beam implantation. Appl. Phys. Express 7, 115201 (2014).

19. Aharonovich, I. \& Neu, E. Diamond Nanophotonics. Adv. Opt. Mater. 2, 911-928 (2014).

20. Wang, C., Ugur, A., Chernyshev, V., Meijer, J. \& Weinfurter, H. in Frontiers in Optics, OSA Technical Digest (CD), JWD106 (Optical Society of America, 2006).

21. Neu, E. et al. Single photon emission from silicon-vacancy colour centres in chemical vapour deposition nano-diamonds on iridium. New J. Phys. 13, 025012 (2011).

22. Neu, E., Agio, M. \& Becher, C. Photophysics of single silicon vacancy centers in diamond: implications for single photon emission. Opt. Express 20, 19956 (2012).

23. Neu, E. et al. Low temperature investigations and surface treatments of colloidal narrowband fluorescent nanodiamonds. J. Appl. Phys. 113, 203507 (2013).

24. Collins, A. T., Allers, L., Wort, C. J. H. \& Scarsbrook, G. A. The annealing of radiation damage in De Beers colourless CVD diamond. Diamond Relat. Mater. 3, 932-935 (1994).

25. Sipahigil, A. et al. Indistinguishable photons from separated silicon-vacancy centers in diamond. Phys. Rev. Lett. 113, 113602 (2014).

26. Hepp, C. et al. Electronic structure of the silicon vacancy color center in diamond. Phys. Rev. Lett. 112, 036405 (2014).

27. Rogers, L. J. et al. Multiple intrinsically identical single-photon emitters in the solid state. Nat. Commun. 5, 4739 (2014).

28. Sipahigil, A. et al. An integrated diamond nanophotonics platform for quantum-optical networks. Science 354, 847-850 (2016).

29. Müller, T. et al. Optical signatures of silicon-vacancy spins in diamond. Nat. Commun. 5, 3328 (2014)

30. Pingault, B. et al. All-optical formation of coherent dark states of silicon-vacancy spins in diamond. Phys. Rev. Lett. 113, 263601 (2014).

31. Rogers, L. J. et al. All-optical initialization, readout, and coherent preparation of single silicon-vacancy spins in diamond. Phys. Rev. Lett. 113, 263602 (2014).

32. Jahnke, K. D. et al. Electron-phonon processes of the silicon-vacancy centre in diamond. New J. Phys. 17, 043011 (2015).

33. D'Haenens-Johansson, U. F. S. et al. Optical properties of the neutral silicon split-vacancy center in diamond. Phys. Rev. B 84, 245208 (2011).

34. Schwartz, J., Michaelides, P., Weis, C. D. \& Schenkel, T. In situ optimization of co-implantation and substrate temperature conditions for nitrogen-vacancy center formation in single-crystal diamonds. New J. Phys. 13, 035022 (2011).

35. Olivero, P. et al. Characterization of three-dimensional microstructures in single-crystal diamond. Diamond Relat. Mater. 15, 1614-1621 (2006).

36. Martin, J., Wannemacher, R., Teichert, J., Bischoff, L. \& Köhler, B. Generation and detection of fluorescent color centers in diamond with submicron resolution. Appl. Phys. Lett. 75, 3096-3098 (1999).

37. Jarmola, A. et al. Longitudinal spin-relaxation in nitrogen-vacancy centers in electron irradiated diamond. Appl. Phys. Lett. 107, 242403 (2015).

38. Koike, J., Parkin, D. M. \& Mitchell, T. E. Displacement threshold energy for type IIa diamond. Appl. Phys. Lett. 60, 1450-1452 (1992).

39. Davies, G., Lawson, S. C., Collins, A. T., Mainwood, A. \& Sharp, S. J. Vacancy-related centers in diamond. Phys. Rev. B 46, 13157-13170 (1992).

40. Evans, R. E., Sipahigil, A., Sukachev, D. D., Zibrov, A. S. \& Lukin, M. D. Narrow-linewidth homogeneous optical emitters in diamond nanostructures via silicon ion implantation. Phys. Rev. Appl. 5, 044010 (2016). 
41. Li, L. et al. Coherent spin control of a nanocavity-enhanced qubit in diamond. Nat. Commun. 6, 6173 (2015).

42. Li, L. et al. Nanofabrication on unconventional substrates using transferred hard masks. Sci. Rep. 5, 7802 (2015).

43. Mouradian, S. L. et al. Scalable integration of long-lived quantum memories into a photonic circuit. Phys. Rev. X 5, 031009 (2015).

44. Abraham, J. B. S., Aguirre, B. A., Pacheco, J. L., Vizkelethy, G. \& Bielejec, E. Fabrication and characterization of a co-planar detector in diamond for low energy single ion implantation. Appl. Phys. Lett. 109, 063502 (2016).

45. Chu, Y. et al. Coherent optical transitions in implanted nitrogen vacancy centers. Nano Lett. 14, 1982-1986 (2014).

46. Iwasaki, T. et al. Germanium-vacancy single color centers in diamond. Sci. Rep. 5, 12882 (2015).

47. Lienhard, B. et al. Bright and photostable single-photon emitter in silicon carbide. Optica 3, 768 (2016).

48. Benjamin, S., Lovett, B. \& Smith, J. Prospects for measurement-based quantum computing with solid state spins. Laser Photon. Rev. 3, 556-574 (2009).

49. Nemoto, K. et al. Photonic architecture for scalable quantum information processing in diamond. Phys. Rev. X 4, 031022 (2014).

50. Kok, P. et al. Linear optical quantum computing with photonic qubits. Rev. Mod. Phys. 79, 135-174 (2007).

51. Li, Y., Humphreys, P. C., Mendoza, G. J. \& Benjamin, S. C. Resource costs for fault-tolerant linear optical quantum computing. Phys. Rev. X 5, 041007 (2015).

52. Azuma, K., Tamaki, K. \& Lo, H.-K. All-photonic quantum repeaters. Nat. Commun. 6, 6787 (2015)

53. Pant, M., Krovi, H., Englund, D. \& Guha, S. Rate-distance tradeoff and resource costs for all-optical quantum repeaters. Phys. Rev. A. 95, 012304 (2017).

54. Gard, B. T., Motes, K. R., Olson, J. P., Rohde, P. P. \& Dowling, J. P. in From Atomic to Mesoscale, 167-192, http://dx.doi.org/10.1142/9789814678704_0008 (2015).

\section{Acknowledgements}

We thank Daniel L. Perry for his assistance in performing the implantation. This research was supported in part by the Army Research Laboratory Center for Distributed Quantum Information (CDQI), the CUA, the NSF, the AFOSR MURI, the Center for Integrated Quantum Materials (NSF grant DMR-1231319) and the DARPA QuINESS programme. Device fabrication was completed via controlled ion implantation with support from the Laboratory Directed Research and Development Program and the Center for Integrated Nanotechnologies, an Office of Science (SC) user facility at Sandia National Laboratories operated for the DOE (contract DE-AC04-94AL85000) by Sandia Corporation, a Lockheed Martin subsidiary.

\section{Author contributions}

T.S., M.E.T. and M.W. carried out post-implantation sample processing, performed optical measurements and analysed the data. L.L. and J.Z. fabricated the diamond cavity sample. M.S. assisted with conversion yield experiments. J.L.P. and R.M.C. performed silicon FIB implantation. A.S., R.E.E., D.D.S. and C.T.N. performed the photoluminescence excitation measurement and the electron co-implantation experiment. T.S., M.E.T., M.W. and D.E. wrote the paper. E.S.B., M.D.L. and D.E. conceived and directed the project. All authors discussed the results and commented on the manuscript.

\section{Additional information}

Competing interests: The authors declare no competing financial interests.

Reprints and permission information is available online at http://npg.nature.com/ reprintsandpermissions/

How to cite this article: Schröder, T. et al. Scalable focused ion beam creation of nearly lifetime-limited single quantum emitters in diamond nanostructures. Nat. Commun. 8, 15376 doi: 10.1038/ncomms15376 (2017)

Publisher's note: Springer Nature remains neutral with regard to jurisdictional claims in published maps and institutional affiliations.

(c) (i) This work is licensed under a Creative Commons Attribution 4.0 International License. The images or other third party material in this article are included in the article's Creative Commons license, unless indicated otherwise in the credit line; if the material is not included under the Creative Commons license, users will need to obtain permission from the license holder to reproduce the material To view a copy of this license, visit http://creativecommons.org/licenses/by/4.0/

C) The Author(s) 2017 